\title{
Long Term Care Leadership Development: New Training for Luke and Yoda
}

\section{Keith $\mathrm{KR}^{*}$}

Department of Health Services, Senior Living Leadership, Bellarmine University, USA

*Corresponding author: Keith Knapp R, Department of Health Services, Senior Living Leadership, Bellarmine University, USA, Email: kknapp@bellarmine.edu

\section{Editorial}

Volume 1 Issue 2

Received Date: July 18, 2017

Published Date: July 28, 2017

DOI: $10.23880 /$ phoa- 16000108

\section{Abstract}

Guidelines vary by state for the length, content and nature of an "Administrator-in-Training" (AIT) experience to qualify for becoming a licensed long term care administrator, as well as for the qualifications and expectations of an AIT's preceptor. A joint effort by the National Association of Long Term Care Administrator Boards (NAB) and American College of Health Care Administrators (ACHCA) has resulted in the introduction of two companion, online training programs to facilitate standardization for both roles, preparing more consistently the next generation of long term care leaders.

\section{Context}

In order to become licensed as a long term care administrator, most states require some degree of experiential learning under the supervision of an already licensed administrator. The length (up to 2,000 hours), content and nature of an "Administrator-in-Training" (AIT) experience varies by state, and many of them have different expectations for applicants with various levels of education and/or work history. Similarly, minimum qualifications and supervision expectations of an AIT's preceptor vary by licensing jurisdiction.

In response to the need for greater uniformity expressed by their respective members, the National Association of Long Term Care Administrator Boards (NAB) [1] and the American College of Health Care Administrators [2] recently introduced two new, webbased training programs.

\section{Approach}

The AIT and Preceptor Programs go hand-in-hand. The AIT Program is a supervised internship, during which the AIT works and trains under a Preceptor's guidance.

Randy Lindner, CEO of NAB, explains, "This unique internship provides an important bridge to work in long term care administration in a Nursing Home, Assisted Living and/or Home and Community-Based Services organization. Research has indicated the importance of professional development for a successful career as a long term care administrator $[3,4] . "$

\section{Goals of the AIT Program}

Working together, the AIT and Preceptor develop the AIT's particular program based on education, experience, knowledge, skills and abilities. 
$\mathrm{NAB}$ and ACHCA recommend that the AIT experience include a minimum of 1,000 hours. (Calculated another way, 1,000 hours would allow the AIT to spend approximately six months in a structured, individualized apprenticeship.) The state licensing board or agency would determine compliance, based on the state's statutory or regulatory requirements.

The National AIT program has three goals:

- Spur and enhance the AIT's professional development in the field of long term care (LTC) administration;

- $\quad$ Equip the AIT with the tools and knowledge needed as an administrator; and

- Engage the AIT in observing and participating in activities associated with administration or resident/client care.

The AIT's experience should complement didactic preparation for the licensing exam, which includes content from each of five practice "domains" (established as essential through a rigorous, periodically updated job analysis): 1) Customer Care, Supports, and Services, 2) Human Resources, 3) Finance, Environment, and 5) Management and Leadership. Online and printed study materials, including the bibliography utilized by NAB for licensure exam development, are available from both $\mathrm{NAB}$ and ACHCA. Ideally, it would include exposure to multiple lines of service.

\section{Goals of On-Line Preceptor Preparation Program}

The Preceptor Preparation Program focuses on four goals:

- Show the basics of how the AIT experiences work;

- Recognize that the Preceptor's relationship with the AIT is more critical to success than any factual information the Preceptor could impart;

- Prepare the Preceptor to lead in building a relationship that embodies mutual respect, trust, honesty and acceptance of risk; and

- Ensure the Preceptor will be both a guide and a role model who facilitates the orientation, development, and professional growth of the AIT.

The Preceptor Preparation Program includes four separate modules - each about an hour long - and is designed to provide a thorough understanding of the AIT experience and expectations for the AIT's Preceptor.

- $\quad$ AIT Experience: structure, roles and responsibilities; attributes of successful Preceptors and AITs; practical strategies to increase the effectiveness of AIT/Preceptor experience.

- Cultivating a Culture of Learning: cultivating a welcoming learning environment; contemporary theories of adult learning; Preceptor in AIT orientation; reconciling ideals learned in school with the practical reality of an administrator's role.

- From Novice to Emerging Leader: assisting an AIT develop professional skills and relationships; verbal and nonverbal communication; guidelines for providing effective feedback; transition from coaching to mentorship.

- Beyond the Domains of Practice: evidence-based practices and fostering critical thinking behaviors; practical examples regarding situations an AIT may encounter (e.g., negotiating a union contract, writing a Plan of Correction, presenting a report to the Board or hiring a new department manager) and modeling problem-resolution, interpersonal communications with various constituents, and participation in professional and civic organizations.

\section{Next Steps}

$\mathrm{NAB}$ recommends to its member boards that all states adopt the NAB/ACHCA AIT Training Program and Preceptor Training Course as their standard requirements. ACHCA advocates that its members adopt the same dual program as best practices to follow.

"We are pleased to offer this training program in partnership with $\mathrm{NAB}$, as our two organizations are committed to excellence in long term care administration," says Cecilia Sepp, President and CEO of ACHCA.

While the training programs are copyrighted, the two organizations are so enthusiastic about the value to the profession that they are offering it for no charge (they just cannot be used as stand-alone revenue generating programs by others). Access both of them by visiting either nabweb.org or achca.org. 


\section{References}

1. NAB, the nation's authority on licensing executives in long term care to deliver quality care, convenes 52 state regulatory boards and agencies, former state regulatory board members, academicians, continuing education sponsors and affiliated associations. It develops, maintains and administers the qualifying examination to become a licensed nursing home administrator; approves continuing education required for renewal; and accredits academic programs in long term care administration.

2. ACHCA is a non-profit professional membership association dedicated to administrative leadership and excellence in post-acute and aging services care across the spectrum of health care services. It offers advanced professional certification, career development opportunities, ongoing education and networking opportunities to its members.

3. Castle NG, Decker F (2011) Top management leadership style and quality of care in nursing homes. Gerontologist 51(5): 630-642.

4. Johs AJ, Olson D, Pratt J (2011) Advancing practices to enhance the field of developing long term care administrators. White Paper supported by The Commonwealth Fund and Foundation of the National Association of Long Term Care Administrator Boards. 